\title{
Research on Energy Optimization Control Technology of Dc Distribution Network
}

\author{
Li Qiuhua, Wang Ping \\ Shandong Huayu University of Technology, Dezhou, Shandong, China
}

Keywords: Energy optimization, Dc distribution network, Control technology

\begin{abstract}
The DC distribution network has the characteristics of good controllability, low loss, high power quality, large transmission capacity, etc., and is very suitable for multi-point access of distributed power sources, so it has received more and more attention and research in recent years. It is a new direction in the development of power distribution systems. Reasonable and effective energy management and optimized operation technology can significantly improve the economics and practicability of DC distribution network, and further promote the development of DC distribution network. Therefore, it is one of the key issues in the development of DC distribution network. Firstly, the characteristics and advantages of DC distribution network are introduced, and the system structure is briefly described. Then the latest research progress of DC distribution network energy optimization management technology and converter instruction coordination control technology based on optimized instruction is carried out. Summary; Finally, the relevant research directions still need to be further improved and improved.
\end{abstract}

\section{Introduction}

In order to meet the needs of sustainable development of humanization, we should reduce human dependence on traditional fossil energy, solve the problem of energy supply shortage, develop and utilize renewable energy, and build a new energy system with high efficiency and sustainable development. The inevitable trend of development. Distributed generation technology has shown great potential in this trend. Distributed generation refers to the use of a variety of available and decentralized energy sources, including renewable energy (solar, biomass, small wind, small hydro, wave energy, etc.) and locally accessible fossil fuels (mainly natural gas). Technology for power generation. On the one hand, distributed generation can use a variety of energy sources to generate electricity, which can help to make full use of clean and renewable energy sources, reduce environmental pollution, and improve the diversity of energy utilization. On the other hand, distributed generation can be used locally. It can save power transmission and transformation and power grid capacity investment, reduce the loss of centralized transmission lines, and can supplement the support of large power grids, improve power quality, and improve the safety and reliability of existing power systems. The large number of grid-connected power generation will not only change the pattern of traditional AC distribution network unidirectional AC power, but also bring severe challenges to the stability and operation technology of traditional AC distribution network: Renewable energy generation in distributed generation (Photovoltaic, wind power, etc.) is characterized by randomness, volatility, unpredictability, etc. It is an uncontrollable distributed power source, and its power generation power fluctuates greatly. A large number of grids will bring severe challenges to the stability of the system. And as the penetration rate of renewable energy generation in the distribution network continues to increase, this situation will become more severe. Distributed power generation is scattered and difficult to control. It is difficult to solve this problem effectively by relying on existing AC power distribution technology. Currently, restrictions and isolation methods are adopted to access distributed power sources, which makes the performance of distributed power supplies. It is difficult to give full play. 


\section{Dc Distribution Network System Structure}

In this paper, the grid-connected power supply and load of the medium voltage DC distribution network are discussed and forecasted. It is pointed out that the design of the DC power distribution system should consider the integration of new technologies such as renewable energy generation, energy storage equipment application and smart grid access. And make full use of the power supply advantages of DC system high level power quality to adapt to the load demand of electricity under different technical conditions. Therefore, in combination with the characteristics and advantages of the above-mentioned DC system, it is known that the DC distribution network will serve as an "embedded" network in the future development to provide power for a region with a large load density or an important and sensitive load in the distribution network. When the system is connected to the grid, the DC output of the AC (converted) interface of the AC (transport) is used as the main power source of the distribution network, and energy exchange can be performed with other AC distribution networks through multiple converter interfaces. There are renewable energy sources such as wind power and photovoltaic power generation, and controllable distributed power sources such as diesel generators and fuel cells, as well as energy storage systems such as energy storage systems and electric vehicle charging (changing) power stations. The flow-sensitive load and the user-side low-voltage DC distribution network and other electrical loads are connected to the grid. When the transmission network or its connected converter station has a short-term failure and the distribution network system is off-grid, in order to ensure the continuous and reliable power supply of the important load, the DC distribution network will be distributed to the system and the energy storage unit. Energy optimization management, increase power output and cut off part of the load and distribution network interconnection interface, so that the system has a certain fault ride-through capability.

The actual medium-voltage DC distribution network, as an intermediate network connecting high-voltage and low-voltage DC systems, should have multiple voltage levels to meet the power requirements of different power supply access and load. Therefore, the voltage level sequence and evaluation system are established for the multi-voltage level DC distribution network, in order to realize the optimal management of system energy, the new energy receiving capacity, power transmission capacity, power supply distance (radius) and electrical insulation protection from the DC distribution network. Reliability assessment and efficient use of new energy, facilitating the increase of its permeability and reducing the economic cost of power electronic equipment in operational control, and comprehensive evaluation and establishment of multi-voltage operating standards; Based on the network topology, a mathematical model is established for the operating trend of the system and the operating characteristics of the power supply and load of the distribution network. The system energy optimization management strategy is formulated according to the different operating states of the system, and a reasonable algorithm is designed to obtain the energy optimization control commands of each grid-connected unit. After obtaining the system energy optimization control instruction, the research on the coordinated control between the system grid-connected unit and each converter and the establishment of the electromagnetic transient simulation model of the system operation will be an important research direction for the future development of the DC distribution network.

\section{Dc Distribution Network Energy Optimization Management}

Summarizing the research results at home and abroad, we can see that there are three main problems in the power flow calculation of multi-terminal DC system considering the commutation process: balance node selection; power flow equation solution; system commutation process equivalent calculation. The equivalent calculation of the commutation process is a special important step in the DC power flow calculation, which is the research difficulty of this problem. It can be known from the existing engineering experience that the transmission system has greater capacity and better stability than the distributed power supply, so it will still be the main source of electrical 
energy for the future DC distribution network. The above characteristics of the transmission network can meet the requirements of the balanced node to maintain the constant bus voltage and flexible output power to compensate for the system energy shortage, so the grid-side commutation output will be the primary choice for the balance node. However, in terms of current engineering applications, due to economic, safety and construction cycle considerations, the current mode of power transmission into the DC system through multiple commutation interfaces is generally adopted. At this time, the dynamic response of the corresponding converter station Characteristics and spare capacity become an important consideration for the selection of balanced nodes.

For the solution of the system power flow equation, due to the existence of distributed power sources such as photovoltaics and wind power in the system and the existence of energy storage equipment, the DC distribution network has the development needs of the two-way flow. At present, the alternating solution method and the unified solution method are two mature methods for solving the power flow calculation problem of multi-terminal flexible DC systems, the former can be effectively compatible with existing AC calculation software, so compared with The latter has certain advantages in terms of algorithm complexity and computational resource occupation; while the latter has obvious advantages in terms of program scalability and computational convergence compared with the former. Based on the alternating solution method, a typical multi-terminal flexible DC system power flow calculation model and mathematical solution method are proposed, which can simultaneously meet the energy interaction between multiple DC systems and AC systems, and the DC network topology is not affected. Limitation, so it will be a good reference for the power flow calculation research of DC distribution network. In terms of commutation loss, the literature believes that the commutation loss can be represented by the constant and variable parts in the mathematical model: the former can be equivalent to the active loss caused by the parallel impedance on the DC distribution line. The node admittance matrix is given a correction, while the latter is proportional to the square of the current on the AC side, which can be equivalent to the impedance increment of the AC side converter transformer. The method can calculate the energy loss of the commutation process more accurately when the DC distribution network is connected to the grid. However, since the system interaction power is unknown and the loss calculation method is complicated at the DC balance node, the calculation amount of the power flow program is greatly increased. Therefore, further improvement and verification are needed. The AC side series resistor $\gamma \mathrm{pu}$ is introduced on this problem to make the AC side loss equivalent. The method directly connects the AC side power output or the load power with the DC side active power, thereby effectively solving the calculation problem of the AC side loss.

Since the energy optimization management problem includes many aspects such as distributed power unit combination, energy storage system operation plan and real-time management of system energy, it is difficult to ensure the economical optimality by considering only the different energy balance relationships at a single time scale. run. Therefore, in recent years, research on system energy optimization management based on multiple time scales has received more and more attention. It is not difficult to find out from the existing research results that the energy optimization management problem of multi-time scale system mainly includes two stages of planning and real-time scheduling. In the planning stage, renewable energy power generation forecasting and system load forecasting are two particularly important research contents, which are directly related to economic indicators such as system power generation cost, power reserve capacity and energy storage system operation plan. At present, the more mature prediction methods can be roughly divided into two categories: power curve prediction and modeling prediction. The latter has received more attention and application in recent years because of its rapid response to actual changes. The modeling types mainly include the following, namely physical modeling, information statistical modeling and learning modeling.

A multi-time scale energy management strategy with two-year optimization structure of real-time planning and real-time scheduling is proposed for the two operating states of grid-connected and off-network. The solar energy reserve capacity of the day-to-day layer is calculated to compensate for the renewable energy generation and load power in the planned future. The prediction error, and 
different optimization objectives are adopted in different situations of grid connection and off-network, so as to realize system energy optimization management. However, the algorithm only determines the energy storage reserve of the current layer based on the predicted capacity of renewable energy, which will inevitably result in waste of energy storage resources. Therefore, how to optimize the energy storage reserve according to the real-time operation of the system to reduce the energy storage capacity requirement is A question worthy of further study. In addition, because the scheduling interval of the scheduling layer is minute, it can not meet the system fluctuation requirements. Therefore, it is worthwhile to add the system energy fluctuation control layer on the shorter time scale to achieve the three-layer coordinated optimization. Further in-depth direction.

\section{Conclusion}

Based on the summary and summary of the research status of DC distribution network, this paper analyzes and studies the operation control technology of DC distribution network. The concept, main tasks and challenges of DC distribution network energy management are analyzed. Based on this, the basic architecture of DC distribution network energy management system is discussed, and a multi-agent energy management system architecture suitable for DC distribution network is proposed. The economic optimization operation model of DC distribution network under multi-time scale is established. The economic operation of the system is realized through coordinated optimization of two stages of planning and real-time scheduling. According to the corresponding optimization objectives and strategies, the model is carried out by using specific examples. The analysis effectively stabilizes the influence of renewable energy uncertainty output and stochastic load demand on system optimization operation; it verifies the correctness and effectiveness of the optimization model.

\section{Acknowledgment}

A Project of Shandong Province Higher Educational Science and Technology Program (J18KA378).

\section{References}

[1] Ma Junchao, Jiang Quanyuan, Yu Peng. Overview of Energy Optimization Control Technology for DC Distribution Network [J]. Automation of Electric Power Systems, 2013(24).

[2] Tu Chunming, Pei Siping, Xiao Fan. Optimization Control Strategy of Three-Port DC Energy Router under TCM Modulation [J]. Power System Technology, 2018, 42(8).

[3] Xu Qingshan, Wu Shengjun, Li Qun. An Optimal Control Strategy for Electric Vehicle Charging Based on DC Microgrid [J]. Transactions of Electrical Engineering, 2016(S2): 116-125.

[4] Ma Xiuda, Kang Xiaoning, Li Shaohua. Design of Hierarchical Control Strategy for Multi-terminal Flexible DC Distribution Network [J]. Journal of Xi'an Jiaotong University, 2016, 50(8): 117-122.

[5] Wang Yi, Zhang Ning, Kang Chongqing. Summary and Prospect of Research on Optimization Planning and Operation of Energy Hub in Energy Internet [J]. Proceedings of the CSEE, 2015, 35(22): 5669-5681. 